University of New Orleans

ScholarWorks@UNO

3-1-1997

\title{
Polarization properties of corner-cube retroreflectors: Theory and experiment
}

Jian Liu

R. M.A. Azzam

University of New Orleans, razzam@uno.edu

Follow this and additional works at: https://scholarworks.uno.edu/ee_facpubs

Part of the Electrical and Electronics Commons

\section{Recommended Citation}

Jian Liu and R. M. A. Azzam, "Polarization properties of corner-cube retroreflectors: theory and experiment," Appl. Opt. 36, 1553-1559 (1997)

This Article is brought to you for free and open access by the Department of Electrical Engineering at ScholarWorks@UNO. It has been accepted for inclusion in Electrical Engineering Faculty Publications by an authorized administrator of ScholarWorks@UNO. For more information, please contact scholarworks@uno.edu. 


\title{
Polarization properties of corner-cube retroreflectors: theory and experiment
}

\author{
Jian Liu and R. M. A. Azzam
}

Polarization properties of the corner-cube retroreflector are discussed theoretically by use of ray tracing and analytical geometry. The Jones matrices and eigenpolarizations of the six propagation trips of the corner-cube retroreflector are derived. An experiment is also set up for the determination of the linear eigenpolarizations and the output states of polarization for incident linearly polarized light. The experimental results are consistent with theoretical expectations. (C) 1997 Optical Society of America

\section{Introduction}

Corner cubes are commonly used as retroreflectors because of their geometrically simple structure, and their polarization properties have been the subject of particular interest. Analysis of corner cubes by Peck, ${ }^{1}$ Rabinowitz et al., ${ }^{2}$ and Lamekin ${ }^{3}$ included a discussion of their eigenpolarization states. Acharekar ${ }^{4}$ derived the internal incidence angles and coordinate transformations between internal reflections for corner cubes. Hodgson and Chipman ${ }^{5}$ presented photographs that qualitatively describe the polarization properties of corner cubes. Mayer ${ }^{6}$ studied the depolarization effects of air-path retroreflectors. Recently Scholl 7 reported on the transformation of the electric field reflected by corner-cube surfaces.

In this paper we present a method, based on solid analytic geometry, for a thorough investigation of light interaction with corner cubes. This includes the internal incidence (or reflection) angles, wave vectors for light propagation in the corner cube, and the coordinate systems for light impinging upon the three internal surfaces. The corner-cube reflector under discussion is a symmetric triangular pyramidlike prism. The bottom face is usually called the base, and the three equal sides form mutually perpendicular internally reflecting surfaces. Light entering the base

When this research was performed, the authors were with the Department of Electrical Engineering, University of New Orleans, New Orleans, Louisiana 70148. J. Liu is now with the Department of Electrical and Computer Engineering, University of Texas at Austin, PRC-MER 1606/R990, Austin, Texas 75758.

Received 15 March 1996; revised manuscript received 3 June 1996.

0003-6935/97/071553-07\$10.00/0

(c) 1997 Optical Society of America at normal incidence is reflected from all three internal surfaces and is returned parallel to the input light beam. In Section 2 reference coordinate systems are established for analyzing light propagation through a corner cube, and one of the six propagation trips is examined in detail. Section 3 gives the Jones matrix and eigenpolarizations for this specific trip. Section 4 provides the Jones matrices for all six propagation trips of the corner cube. Experimental results are presented in Section 5 for verifying the theoretical analysis. Section 6 gives the conclusion.

\section{Analytic Geometry for Corner-Cube Retroreflector}

To simplify ray tracing, the corner cube is represented as shown in Fig. 1. The three right-angle isosceles triangles $A B O, B C O$, and $C A O$ are the internal reflecting surfaces of the corner cube, and are located in the $X Y, X Z$, and $Y Z$ planes, respectively. Equilateral triangle $A B C$ is the base. Let $O A=$ $O B=O C=a$. The equations of the four surfaces and their unit normal vectors are

$$
X=0, \quad \mathbf{N}_{1}=\mathbf{i}
$$

for surface $A C O$;

$$
Z=0, \quad \mathbf{N}_{2}=-\mathbf{k}
$$

for surface $A B O$;

$$
Y=0, \quad \mathbf{N}_{3}=-\mathbf{j}
$$

for surface $B C O$;

$$
\begin{gathered}
X-Y-Z+a=0, \\
\mathbf{N}_{4}=(-\mathbf{i}+\mathbf{j}+\mathbf{k}) / 3^{1 / 2} ;
\end{gathered}
$$




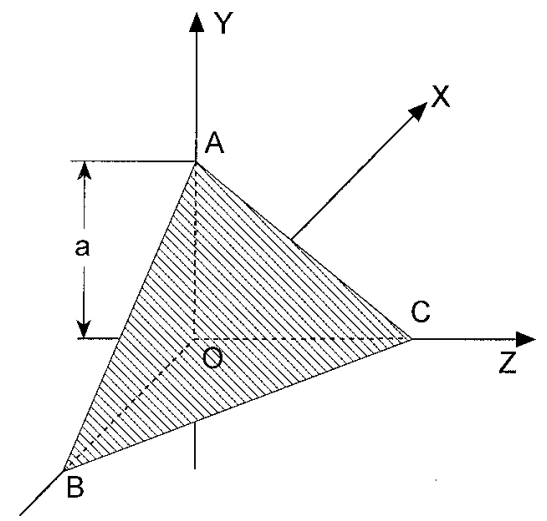

Fig. 1. Geometry of the corner-cube retroreflector.

for surface $A B C$, where $\mathbf{i}, \mathbf{j}$, and $\mathbf{k}$ are unit vectors along the coordinate axes $X, Y$, and $Z$, respectively.

We assume that the light beam enters the cube in a direction opposite to $\mathbf{N}_{4}$, normal to the base surface of the corner cube, and is reflected totally from the internal surfaces in the order of $A C O, A B O$, and $B C O$; i.e., light enters the cube from the left-hand side and emerges after a counterclockwise trip from the cube in the same direction of $\mathbf{N}_{4}$. This is one of the six distinct trips of light propagating in the corner-cube retroreflector.

For specificity, let us take $a=10 \mathrm{~cm}$ and the coordinates of the point of light impinging upon the base surface to be $O_{1}(-2.5000,4.0000,3.5000)$. The external reference $p$-polarization direction for the input and the output light is defined as perpendicular to side $A B$, and the $s$-polarization direction is parallel to it, as shown in Fig. 2. Light propagation within the corner cube is examined by ray tracing and analytic geometry. The sign conventions are consistent with those discussed by Azzam and Bashara. ${ }^{8}$ The normal to the plane of incidence for light reflection at each surface of the cube is defined as parallel to the local $s$-polarization direction. The unit vectors along the incident and reflected light from each internal

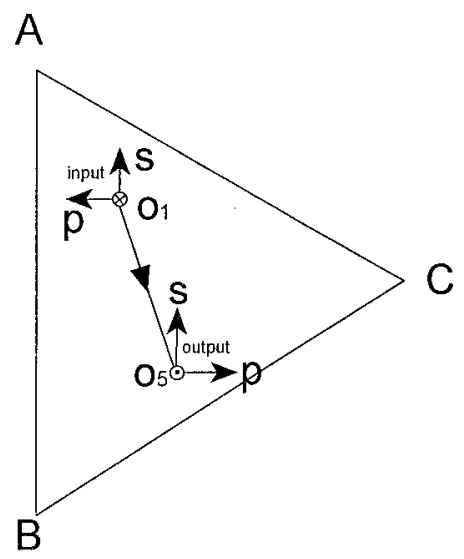

Fig. 2. Definition of the external reference $p$ - and $s$-polarization directions for the input and the output light. Edge $A B$ of the base of the corner cube is used as a reference.

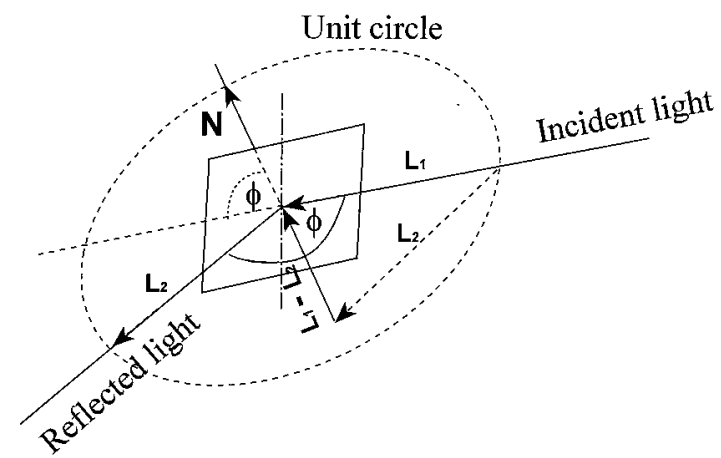

Fig. 3. Propagation vectors for the incident and reflected light from a planer surface. $\mathbf{N}$ is the surface normal.

surface are related by ${ }^{9}$

$$
\mathbf{L}_{2}=\mathbf{L}_{1}-2 \mathbf{N} \cos \phi,
$$

where $\mathbf{L}_{1}$ is the unit vector along the incident ray and $\mathbf{L}_{2}$ is the unit vector along the reflected ray, as shown in Fig. 3. The corresponding unit normal $\mathbf{n}$ to the plane of incidence is given by

$$
\mathbf{n}=\mathbf{L}_{1} \times \mathbf{L}_{2} /\left|\mathbf{L}_{1} \times \mathbf{L}_{2}\right|
$$

for the counterclockwise propagation trip.

The angle of incidence $\phi$ for each internal surface is

$$
\phi=\cos ^{-1}\left(\frac{1}{\sqrt{3}}\right)=54.7356^{\circ}
$$

for light normally incident upon the base surface of the corner-cube retroflector. If the incident light deviates from the normal, the angle of incidence will be different at each surface.

The position coordinates of the points where light impinges upon the surfaces of the corner cube are listed in Table 1. For this case the displacement between the input and the output light is $O_{1} O_{5}=$ $2.1602 \mathrm{~cm}$. The unit vectors along the round-trip segmented light path, as shown in Fig. 4, are listed in Table 2. The unit vector along $O_{1} O_{5}$ is given by

$$
\mathbf{L}_{6}=\mathbf{O}_{1} \mathbf{O}_{5} / O_{1} O_{5}=(-0.7715,-0.6172,-0.1543) \text {. }
$$

The unit normal to the plane defined by the input and output light rays is given by

$$
\mathbf{n}_{6}=\frac{\mathbf{L}_{0} \times \mathbf{L}_{6}}{\left|\mathbf{L}_{0} \times \mathbf{L}_{6}\right|}=(-0.2673,0.5345,-0.8018) .
$$

Table 1. Coordinates of Points of Intersection with Each Surface of a Corner Cube, $a=10 \mathrm{~cm}$

\begin{tabular}{ll}
\hline \multicolumn{1}{c}{ Surface } & \multicolumn{1}{c}{ Intersection Coordinates } \\
\hline Base (input) & $O_{1}(-2.5000,4.0000,3.5000)$ \\
$A C O$ & $O_{2}(0.0000,1.5000,1.0000)$ \\
$A B O$ & $O_{3}(-1.000,0.5000,0.0000)$ \\
$B C O$ & $O_{4}(-1.500,0.0000,0.5000)$ \\
Base (output) & $O_{5}(-4.1667,2.6667,3.1667)$ \\
\hline
\end{tabular}




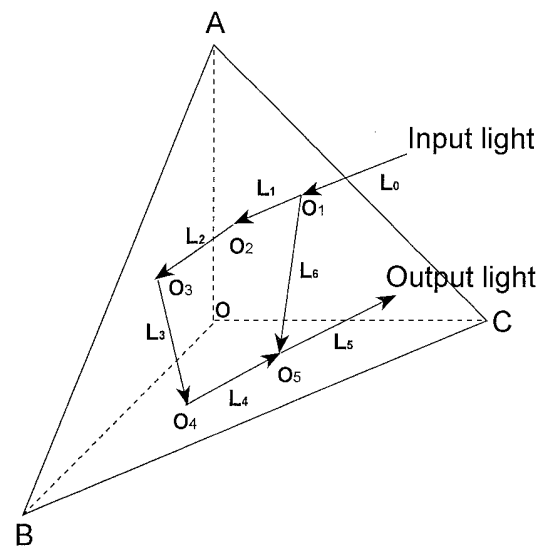

Fig. 4. Light ray vectors for the $A C O-A B O-B C O$ propagation trip.

The external reference $s$-polarization direction for the input light shown in Fig. 2 is given by

$$
\mathbf{n}_{1}=(0.7071,0.7071,0.0000) .
$$

The normal of the incidence plane for surface $A C O$ is

$$
\mathbf{n}_{2}=\frac{\mathbf{L}_{1} \times \mathbf{L}_{2}}{\left|\mathbf{L}_{1} \times \mathbf{L}_{2}\right|}=(0.0000,0.7071,-0.7071)
$$

The normal of the incidence plane for surface $A B O$ is

$$
\mathbf{n}_{3}=\frac{\mathbf{L}_{2} \times \mathbf{L}_{3}}{\left|\mathbf{L}_{2} \times \mathbf{L}_{3}\right|}=(-0.7071,0.7071,0.0000) \text {. }
$$

The normal of the incidence plane for surface $B C O$ is

$$
\mathbf{n}_{4}=\frac{\mathbf{L}_{3} \times \mathbf{L}_{4}}{\left|\mathbf{L}_{3} \times \mathbf{L}_{4}\right|}=(-0.7071,0.0000,-0.7071) .
$$

The external reference $s$-polarization direction for the output light is the same as that for the input light (Fig. 2):

$$
\mathbf{n}_{5}=(0.7071,0.7071,0.0000) .
$$

The angles between the normals of the incidence planes for the surfaces of the corner cube and $\mathbf{n}_{1}$ are listed in Table 3. The angles between the normals of the incidence planes for the surfaces of the corner cube and the unit normal $\mathbf{n}_{6}$ to the plane defined by the input and output light directions are listed in Table 4 for reference.

It is also important to find the angle between the normals of the successive incidence planes, which

Table 2. Unit Vectors Along the Segmented Light Path

\begin{tabular}{cl}
\hline Unit Vector & \multicolumn{1}{c}{ Coordinate } \\
\hline $\mathbf{L}_{0}$ & $(0.5774,-0.5774,-0.5774)$ \\
$\mathbf{L}_{1}$ & $(0.5774,-0.5774,-0.5774)$ \\
$\mathbf{L}_{2}$ & $(-0.5774,-0.5774,-0.5774)$ \\
$\mathbf{L}_{3}$ & $(-0.5774,-0.5774,0.5774)$ \\
$\mathbf{L}_{4}$ & $(-0.5774,0.5774,0.5774)$ \\
$\mathbf{L}_{5}$ & $(-0.5774,0.5774,0.5774)$ \\
\hline
\end{tabular}

Table 3. Angles Between the Surface Normals and $n_{1}$

\begin{tabular}{cccr}
\hline $\begin{array}{c}\text { Surface } \\
\text { Normal }\end{array}$ & Angle & $\begin{array}{c}\text { Surface } \\
\text { Normal }\end{array}$ & Angle \\
\hline $\mathbf{n}_{2}$ & 60.0000 & $\mathbf{n}_{4}$ & 120.0000 \\
$\mathbf{n}_{3}$ & 90.0000 & $\mathbf{n}_{5}$ & 0.0000 \\
\hline
\end{tabular}

define the angle of rotation of the coordinate transformation Jones matrix. By following the sign conventions used above ${ }^{8}$ and looking against the direction of light propagation, we obtain the relative angles of rotation listed in Table 5.

\section{Jones Matrix of the Propagation Trip $A C O \rightarrow A B O$} $\rightarrow \mathrm{BCO}$

The reflection Jones matrix for each surface of the corner cube takes the form ${ }^{8}$

$$
T_{i}=\left[\begin{array}{cc}
\left|R_{p_{i}}\right| \exp \left(j \delta_{p_{i}}\right) & 0 \\
0 & \left|R_{s_{i}}\right| \exp \left(j \delta_{s_{i}}\right)
\end{array}\right], \quad i=1,2,3
$$

For convenience, we use the simpler notation

$$
T_{i}=\left[\begin{array}{cc}
r_{p_{i}} & 0 \\
0 & r_{s_{i}}
\end{array}\right], \quad i=1,2,3
$$

where subscript 1 represents surface $A C O ; 2, A B O$; and $3, B C O$.

Then the overall Jones matrix of the $A C O \rightarrow A B O$ $\rightarrow B C O$ trip for the corner-cube retroreflecter relative to the input and output ps coordinate systems as shown in Fig. 2 can be written as

$$
J_{123}=R\left(-120^{\circ}\right) T_{3} R\left(60^{\circ}\right) T_{2} R\left(-60^{\circ}\right) T_{1} R\left(-60^{\circ}\right),
$$

where the rotation matrix is defined by

$$
R(\theta)=\left[\begin{array}{cc}
\cos \theta & \sin \theta \\
-\sin \theta & \cos \theta
\end{array}\right]
$$

Table 4. Angles for Surface Normals Relative to $n_{6}$

\begin{tabular}{cccc}
\hline $\begin{array}{c}\text { Surface } \\
\text { Normal }\end{array}$ & Angle & $\begin{array}{c}\text { Surface } \\
\text { Normal }\end{array}$ & Angle \\
\hline $\mathbf{n}_{1}$ & 79.1066 & $\mathbf{n}_{4}$ & 40.8934 \\
$\mathbf{n}_{2}$ & 19.1066 & $\mathbf{n}_{5}$ & 79.1066 \\
$\mathbf{n}_{3}$ & 55.4624 & & \\
\hline
\end{tabular}

Table 5. Angles of Rotation of Coordinates (deg)

\begin{tabular}{ccr}
\hline Normals & Rotation & \multicolumn{1}{c}{ Angle } \\
\hline $\mathbf{n}_{1}, \mathbf{n}_{2}$ & $\alpha_{1}$ & -60.0000 \\
$\mathbf{n}_{2}, \mathbf{n}_{3}$ & $\alpha_{2}$ & -60.0000 \\
$\mathbf{n}_{3}, \mathbf{n}_{4}$ & $\alpha_{3}$ & 60.0000 \\
$\mathbf{n}_{4}, \mathbf{n}_{5}$ & $\alpha_{4}$ & -120.0000 \\
\hline
\end{tabular}


Substitution of the individual rotation and reflection matrices into Eq. (18) gives

$$
J_{123}=\left[\begin{array}{ll}
J_{11} & J_{12} \\
J_{21} & J_{22}
\end{array}\right],
$$

where

$$
\begin{aligned}
J_{11}= & r_{p_{2}}\left(r_{p_{1}} \cos ^{2} 60^{\circ}-r_{s_{1}} \sin ^{2} 60^{\circ}\right)\left(-r_{p_{3}} \cos ^{2} 60^{\circ}\right. \\
& \left.+r_{s_{3}} \sin ^{2} 60^{\circ}\right)-\left(r_{p_{1}}+r_{s_{1}}\right) \\
& \times\left(r_{p_{3}}+r_{s_{3}}\right) r_{s_{2}} \sin ^{2} 60^{\circ} \cos ^{2} 60^{\circ}, \\
J_{12}= & r_{p_{2}}\left(r_{p_{1}}+r_{s_{1}}\right)\left(r_{p_{3}} \cos ^{2} 60^{\circ}-r_{s_{3}} \sin ^{2} 60^{\circ}\right) \\
& \times \sin 60^{\circ} \cos 60^{\circ}-\left(-r_{p_{1}} \sin ^{2} 60^{\circ}\right. \\
& \left.+r_{s_{1}} \cos ^{2} 60^{\circ}\right)\left(r_{p_{3}}+r_{s_{3}}\right) r_{s_{2}} \sin 60^{\circ} \cos 60^{\circ},
\end{aligned}
$$

$$
\begin{aligned}
J_{21}= & \left(-r_{s_{1}} \sin ^{2} 60^{\circ}+r_{p_{1}} \cos ^{2} 60^{\circ}\right) \\
& \times\left(r_{p_{3}}+r_{s_{3}}\right) r_{p_{2}} \sin 60^{\circ} \cos 60^{\circ}-\left(-r_{p_{3}} \sin ^{2} 60^{\circ}\right. \\
& \left.+r_{s_{3}} \cos ^{2} 60^{\circ}\right)\left(r_{p_{1}}+r_{s_{1}}\right) r_{s_{2}} \sin 60^{\circ} \cos 60^{\circ},
\end{aligned}
$$

$$
\begin{aligned}
J_{22}= & r_{s_{2}}\left(r_{s_{1}} \cos ^{2} 60^{\circ}-r_{p_{1}} \sin ^{2} 60^{\circ}\right)\left(-r_{s_{3}} \cos ^{2} 60^{\circ}\right. \\
& \left.+r_{p_{3}} \sin ^{2} 60^{\circ}\right)-\left(r_{p_{1}}+r_{s_{1}}\right) \\
& \times\left(r_{p_{3}}+r_{s_{3}}\right) r_{p_{2}} \sin ^{2} 60^{\circ} \cos ^{2} 60^{\circ} .
\end{aligned}
$$

If the optical properties for the three internal surfaces are assumed to be identical and the incident light is normal to the base surface of the corner-cube reflector, the reflection coefficients are the same for three internal surfaces. The elements for the Jones matrix in Eqs. (21) then become

$$
J_{11}=-\frac{1}{16}\left(r_{p}^{3}+15 r_{p} r_{s}^{2}-3 r_{s} r_{p}^{2}+3 r_{s}^{3}\right),
$$

$$
\begin{aligned}
& J_{12}=\frac{\sqrt{3}}{16}\left(r_{p}{ }^{3}+r_{s} r_{p}{ }^{2}-r_{p} r_{s}{ }^{2}-r_{s}{ }^{3}\right), \\
& J_{21}=\frac{\sqrt{3}}{16}\left(r_{p}{ }^{3}+r_{s} r_{p}{ }^{2}-r_{p} r_{s}{ }^{2}-r_{s}{ }^{3}\right),
\end{aligned}
$$

$$
J_{22}=-\frac{1}{16}\left(3 r_{p}^{3}-3 r_{p} r_{s}^{2}+15 r_{s} r_{p}^{2}+r_{s}^{3}\right) .
$$

For a solid glass corner cube with refractive index 1.515 , total internal reflection occurs at the three internal surfaces. The reflection phase shifts are $\delta_{p}$ $=124.7455^{\circ}$ and $\delta_{s}=79.5499^{\circ}$. The Jones matrix of the $A C O \rightarrow A B O \rightarrow B C O$ trip calculated by use of Eqs. (22) is

$$
J_{123}=\left[\begin{array}{cc}
-0.0266+0.9586 i & 0.2282+0.1685 i \\
0.2282+0.1685 i & -0.9083+0.3075 i
\end{array}\right] .
$$

The Jones eigenvectors ${ }^{8}$ of the $A C O \rightarrow A B O \rightarrow B C O$ trip for the corner cube reflector are

$$
E_{e 1}=\left[\begin{array}{c}
1 \\
0.2434
\end{array}\right], \quad E_{e 2}=\left[\begin{array}{c}
1 \\
-4.1076
\end{array}\right],
$$

which represent orthogonal linear polarization states. The corresponding complex eigenvalues are

$$
\begin{aligned}
& V_{e 1}=0.0290+0.9996 i, \\
& V_{e 2}=-0.9638+0.2665 i .
\end{aligned}
$$

\section{Jones Matrices and Eigenpolarizations of the Six Propagation Trips}

The six propagation trips are divided into two groups: the three counterclockwise propagation trips $A C O \rightarrow$ $A B O \rightarrow B C O, A B O \rightarrow B C O \rightarrow A C O$, and $B C O \rightarrow$ $A C O \rightarrow A B O$, with the unit normal $\mathbf{n}$ to the plane of incidence given by Eq. (7); the other set consists of the

Table 6. Jones Matrices, Eigenvectors, and Eigenvalues for the Counterclockwise Propagation Trips of a Corner-Cube Retroreflector

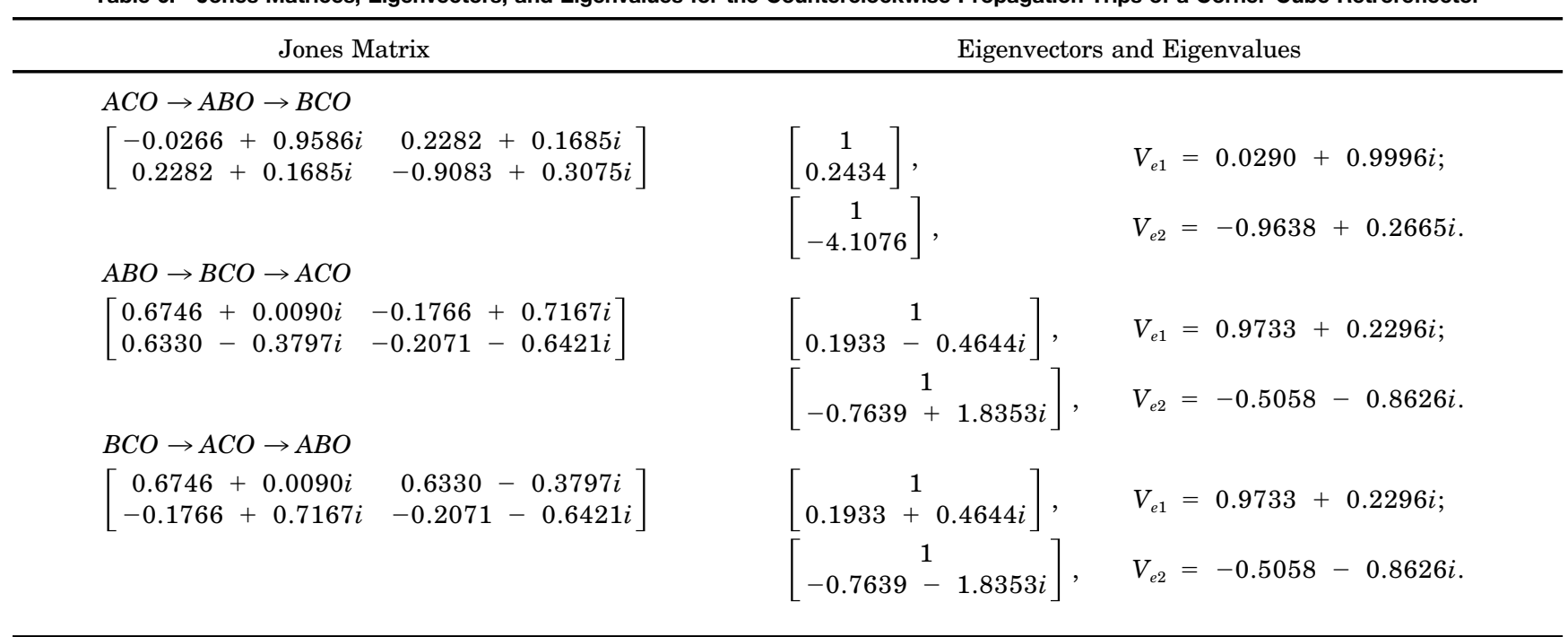




$$
\begin{aligned}
& B C O \rightarrow A B O \rightarrow A C O \\
& {\left[\begin{array}{cc}
-0.0266+0.9586 i & -0.2282-0.1685 i \\
-0.2282-0.1685 i & -0.9083+0.3075 i
\end{array}\right]} \\
& A C O \rightarrow B C O \rightarrow A B O \\
& {\left[\begin{array}{cc}
0.6746+0.0090 i & -0.6330+0.3797 i \\
0.1766-0.7167 i & -0.2071-0.6421 i
\end{array}\right]} \\
& A B O \rightarrow A C O \rightarrow B C O \\
& {\left[\begin{array}{cc}
0.6746+0.0090 i & 0.1766-0.7167 i \\
-0.6330+0.3797 i & -0.2071-0.6421 i
\end{array}\right]}
\end{aligned}
$$

$$
\begin{aligned}
& {\left[\begin{array}{c}
1 \\
-0.2434
\end{array}\right],} \\
& {\left[\begin{array}{c}
1 \\
4.1076
\end{array}\right],}
\end{aligned}
$$$$
\left[\begin{array}{cc}
1 & \\
-0.1933 & -0.4644 i
\end{array}\right]
$$$$
\left[\begin{array}{c}
1 \\
0.7639+1.8353 i
\end{array}\right] \text {, }
$$$$
\left[\begin{array}{c}
1 \\
-0.1933+0.4644 i
\end{array}\right],
$$$$
\left[\begin{array}{c}
1 \\
0.7639-1.8353 i
\end{array}\right] \text {, }
$$

$$
\begin{aligned}
& V_{e 1}=0.0290+0.9996 i ; \\
& V_{e 2}=-0.9638+0.2665 i .
\end{aligned}
$$$$
V_{e 1}=0.9733+0.2296 i ;
$$$$
V_{e 2}=-0.5058-0.8626 i .
$$$$
V_{e 1}=0.9733+0.2296 i ;
$$$$
V_{e 2}=-0.5058-0.8626 i .
$$

clockwise propagation trips with

$$
\mathbf{n}^{\prime}=-\mathbf{L}_{1} \times \mathbf{L}_{2} /\left|\mathbf{L}_{1} \times \mathbf{L}_{2}\right| .
$$

for the trips $A C O \rightarrow B C O \rightarrow A B O, B C O \rightarrow A B O \rightarrow$ $A C O$, and $A B O \rightarrow A C O \rightarrow B C O$.

Following the sign conventions and coordinate system used above, we obtain the relative angles between the normals of the successive incidence planes for each propagation trip, which define the angles of rotation of the coordinate transformations. Then the overall Jones matrix for the corner-cube retroreflecter can be written as

$$
\begin{aligned}
& J_{123}=R\left(-120^{\circ}\right) T_{3} R\left(60^{\circ}\right) T_{2} R\left(-60^{\circ}\right) T_{1} R\left(-60^{\circ}\right), \\
& J_{231}=R\left(120^{\circ}\right) T_{1} R\left(60^{\circ}\right) T_{3} R\left(-60^{\circ}\right) T_{2} R\left(180^{\circ}\right), \\
& J_{312}=R\left(0^{\circ}\right) T_{2} R\left(60^{\circ}\right) T_{1} R\left(-60^{\circ}\right) T_{3} R\left(60^{\circ}\right), \\
& J_{321}=R\left(-60^{\circ}\right) T_{1} R\left(-60^{\circ}\right) T_{2} R\left(60^{\circ}\right) T_{3} R\left(-120^{\circ}\right), \\
& J_{132}=R\left(180^{\circ}\right) T_{2} R\left(-60^{\circ}\right) T_{3} R\left(60^{\circ}\right) T_{1} R\left(120^{\circ}\right), \\
& J_{213}=R\left(60^{\circ}\right) T_{3} R\left(-60^{\circ}\right) T_{1} R\left(60^{\circ}\right) T_{2} R\left(0^{\circ}\right),
\end{aligned}
$$

where the subscript notation is already explained in Section 3.

For the specific corner cube described in Section 3, the Jones matrices and eigenpolarizations for the six propagation trips are calculated by use of Eqs. (27)(32) and are listed in Tables 6 and 7 . We note that two of the six propagation trips have linear eigenpolarizations and that the other four propagation trips have elliptical eigenpolarizations. It should be emphasized here that the directions of the $p$ and the $s$ polarizations of the input and the output light are referred to the edge $A B$ of the corner cube (Fig. 2) and that the convention of analyzing polarized light assumes that one looks against the light propagation direction. That same reference system is used for all six propagation trips. Other authors presented Jones matrices for the corner-cube retroreflector based on different choices of the reference coordinate system, and different coordinate systems for different trips. ${ }^{1,2}$ The dependence of eigenpolarizations on the choice of coordinate system is discussed in Appendix A.

\section{Experimental Results}

\section{A. Determination of the Linear Eigenpolarizations}

An experiment was set up for verification of the linear eigenpolarizations of the corner cube. In Fig. 5 the light beam emitted from a He-Ne laser of wavelength $623.8 \mathrm{~nm}$ passes through a crystal polarizer and is retroreflected from a BK7 glass corner-cube retroreflector ${ }^{10}$ with refractive index 1.515 (at $\lambda=632.8 \mathrm{~nm}$ ) in the 123 light propagation trip as discussed above. The polarizer is aligned so that the transmitted polarization with $0^{\circ}$ azimuth is perpendicular to side $A B$ and parallel to the base $A B C$ of the corner cube. The output light from the corner cube is then reflected at near-normal incidence by a mirror, passes through an analyzer, and is received by a photodetector. The input and output light beams are aligned parallel to the axis of the corner cube. With the polarization azimuth set at one of the calculated eigenpolarizations, the extinction position of the analyzer is determined. The polarizer is then rotated $90^{\circ}$ to yield the orthogonal eigenpolarization, and the corresponding ex-

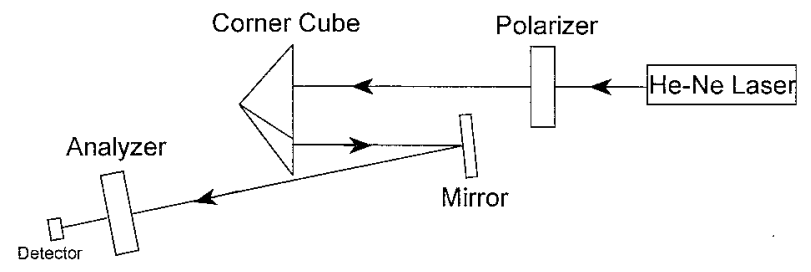

Fig. 5. Experimental setup for the determination of the linear eigenpolarizations of a corner-cube retroreflector. 
Table 8. Experimental Results for the Determination of the Linear Eigenpolarizations of a Corner Cube

\begin{tabular}{cccc}
\hline $\begin{array}{c}\text { Azimuth for } \\
\text { Input }\end{array}$ & $\begin{array}{c}\text { Polarizer } \\
\text { Azimuth } \\
\text { Eigenpolarization }\end{array}$ & $\begin{array}{c}\text { Analyzer } \\
\text { Azimuth, } \\
90^{\circ}(\mathrm{deg})\end{array}$ & $\mathscr{L}$ \\
\hline 13.68 & 13.77 & 13.18 & 0.9998 \\
103.68 & 103.38 & 102.72 & 1.0000 \\
$13.68+180$ & 193.65 & 192.80 & 0.9999 \\
$103.68+180$ & 283.52 & 283.39 & 0.9999 \\
\hline
\end{tabular}

tinction position of the analyzer is also measured. During the experiment fine adjustments of both the polarizer and analyzer are made, to yield maximum extinction.

To represent the purity of linear polarization, we introduce a parameter $\mathscr{L}$ defined by

$$
\mathscr{L}=\left(V_{\max }-V_{\min }\right) /\left(V_{\max }+V_{\min }\right),
$$

where $V_{\max }$ and $V_{\min }$ are the maximum and minimum output voltages of the detector located behind the analyzer. For an ideal linear state of polarization, $\mathscr{L}=1$. The experimental results for the $A C O$ $\rightarrow A B O \rightarrow B C O$ propagation trip, given in Table 8, confirm that the eigenpolarizations of the corner-cube retroreflector are indeed orthogonal linear states as predicted by the theory of Section 3. The experimental results for the reverse trip $B C O \rightarrow A B O \rightarrow$ $A C O$ also confirm the linear eigenpolarization prediction.

\section{B. Output State of Polarization for Input Linear Polarizations}

As shown in Fig. 6, two liquid crystal (LC) retarders ${ }^{11}$ are aligned in such a way that the fast axis of the first has an azimuth of $45^{\circ}$ and that of the second $0^{\circ}$. The analyzer transmission axis is perpendicular to the fast axis of the first LC retarder. A photodetector is placed behind the analyzer. The output signal of the detector is nulled by adjustment of the voltages of the two LC retarders, and the state of polarization of the input light (taking into consideration the half-wave retardation effect of the mirror that directs the output light to the detector) is determined by the Jones vector ${ }^{8}$

$$
E=\left[\begin{array}{c}
1 \\
-X
\end{array}\right]
$$

where

$$
X=\frac{i \sin \frac{\delta_{1}}{2}-\cos \frac{\delta_{1}}{2} \exp \left(i \delta_{2}\right)}{\cos \frac{\delta_{1}}{2}-i \sin \frac{\delta_{1}}{2} \exp \left(i \delta_{2}\right)} .
$$

The corresponding normalized Stokes parameters can be easily calculated. ${ }^{8}$

The light emitted from a He-Ne laser with wavelength $632.8 \mathrm{~nm}$ and $8 \mathrm{~mW}$ of output power passes through a crystal polarizer that rotates from $0^{\circ}$ to
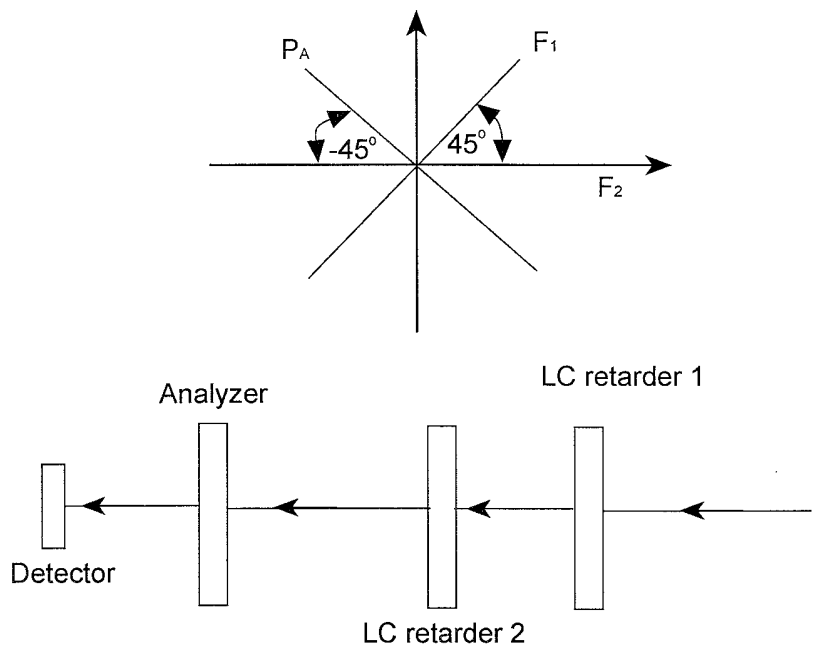

Fig. 6. Polarization-state detector that uses two liquid crystal (LC) retarders and an analyzer. $\quad F_{1}$ is the fast axis for LC retarder $1 ; \mathrm{F}_{2}$ is the fast axis for $\mathrm{LC}$ retarder 2 ; and $\mathrm{P}_{A}$ is the transmission axis of the analyzer.
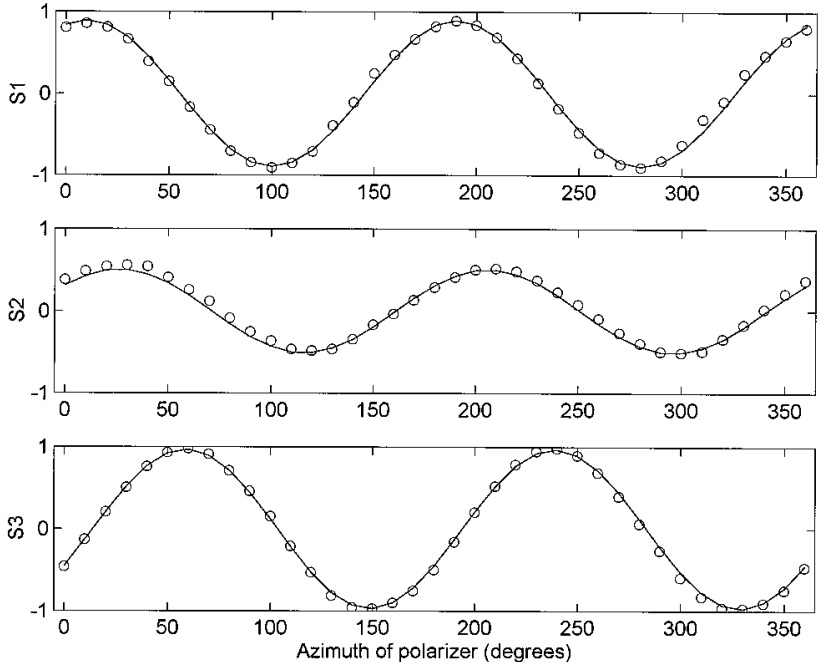

Fig. 7. Measured normalized Stokes parameters of the output light of a corner cube when the incident light is linearly polarized. The propagation trip is $A C O \rightarrow A B O \rightarrow B C O$. Solid curves are theoretically calculated from Eq. (23) and the circles are experimental results.

$360^{\circ}$ in steps of $10^{\circ}$. The linearly polarized output light from this polarizer goes through the $A C O \rightarrow$ $A B O \rightarrow B C O$ propagation trip and is received by the polarization state detector that consists of the two LC retarders and the analyzer as discussed above. Theoretical results (solid curves) determined from the Jones matrix of Eq. (23) and experimental ones (circles) are in good agreement as shown in Fig. 7.

Experiments were also carried out for all the other propagation trips. The results are again consistent with the theoretical predictions. Figure 8 gives another example of the $A C O \rightarrow B C O \rightarrow A B O$ propagation trip with the Jones matrix given by Eq. (31). 

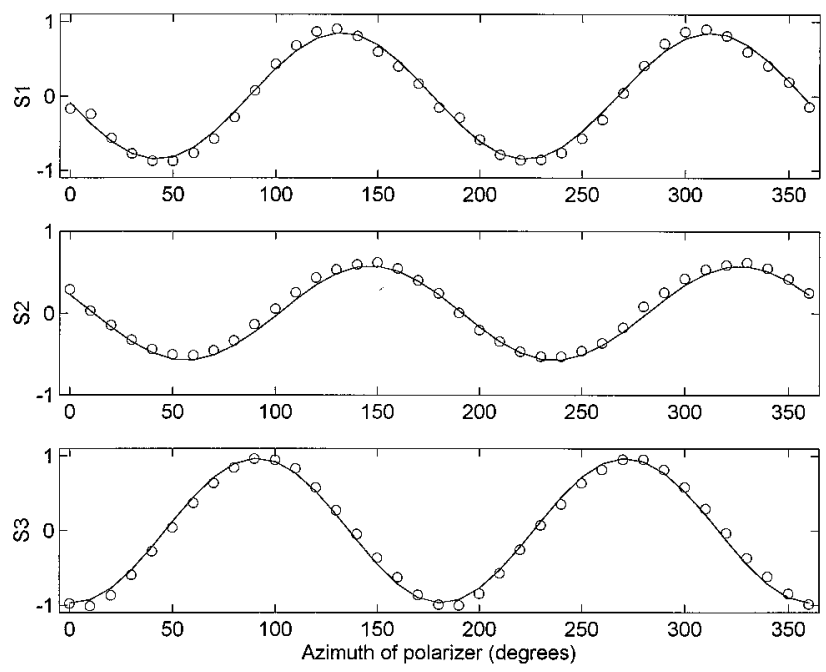

Fig. 8. Measured normalized Stokes parameters of the output light of a corner cube when the incident light is linearly polarized. The propagation trip is $A C O \rightarrow B C O \rightarrow A B O$. Solid curves are theoretically calculated from Eq. (31) and the circles are experimental results.

\section{Conclusion}

We have demonstrated, both in theory and experiment, that if the reference coordinate systems for the input and output light are selected with respect to one edge of the base of a corner cube, orthogonal linear eigenpolarizations are obtained for the $A C O \rightarrow$ $A B O \rightarrow B C O$ propagation trip and its reverse propagation trip, whereas elliptical eigenpolarizations are obtained for the other four trips.

\section{Appendix A: Dependence of Eigenpolarizations on the Choice of Input and Output Coordinate Systems}

The Jones matrix of an optical system depends on the choice of the reference transverse coordinate systems that are used in the input and output beams. ${ }^{8}$ Consequently, the eigenpolarizations also depend on such a choice, except when the optical system has orthogonal circular eigenpolarization states.

To take a simple example, let us consider light reflection by an optically isotropic plane mirror. If the input and the output coordinate systems are chosen to coincide with the directions parallel $(p)$ and perpendicular $(s)$ to the plane of incidence in the incident and the reflected beams, the reflection Jones matrix $M$ is diagonal and the eigenpolarizations are the $p$ and $s$ linear states. Now suppose that only the output coordinate system is rotated by an angle $\alpha \neq$ $0,90^{\circ}$ to the directions $p^{\prime}$ and $s^{\prime}$, whereas the input system remains unchanged. In this case the new Jones matrix is given by $R(\alpha) M$, where $R(\alpha)$ is the $2 \times$ 2 rotation matrix. The new Jones matrix is no longer diagonal and its eigenvectors indicate apparent "elliptical eigenpolarizations". The latter represent two invariant-ellipticity states ${ }^{12}$ that experience a rotation $\alpha$ on reflection. Changing the angle $\alpha$ generates an infinite number of such pairs of invariant-ellipticity states whose loci are described by Azzam and Bashara. ${ }^{12,13}$

Jian Liu would like to thank Yifeng Cui for helpful discussion on the experimental setup.

\section{References}

1. E. R. Peck, "Polarization properties of corner reflectors and cavities," J. Opt. Soc. Am. 52, 253-257 (1962).

2. P. Rabinowitz, S. F. Jacobs, T. Shultz, and G. Gould, "Cubecorner Fabry-Perot interferometer," J. Opt. Soc. Am. 52, 452453 (1962).

3. P. I. Lamekin, "Intrinsic polarization states of corner reflectors," Sov. J. Opt. Tech. 54, 658-661 (1987).

4. M. A. Acharekar, "Derivation of internal incidence angles and coordinate transformations between internal reflections for corner reflectors at normal incidence," Opt. Eng. 23, 669-674 (1984).

5. R. R. Hodgson and R. A. Chipman, "Measurement of corner cube polarization," in Polarimetry: Radar, Infrared, Visible, Ultraviolet, and X-Ray, R. A. Chipman and J. W. Morris, eds., Proc. SPIE 1317, 436-447 (1990).

6. J. R. Mayer, "Polarization optics design for a laser tracking triangulation instrument based on dual-axis scanning and a retroreflective target," Opt. Eng. 32, 3316-3326 (1993).

7. M. S. Scholl, "Ray trace through a corner-cube retroreflector with complex reflection coefficients," J. Opt. Soc. Am. A 12, 1589-1592 (1995).

8. R. M. A. Azzam and N. M. Bashara, Ellipsometry and Polarized Light (North-Holland, Amsterdam, 1977).

9. D. Korsch, Reflective Optics (Academic, New York, 1991).

10. Edmund Scientific, Barrington, New Jersey.

11. Meadowlark Optics, Longmont, Colorado.

12. R. M. A. Azzam and N. M. Bashara, "Loci of invariant-azimuth and invariant-ellipticity polarization states of an optical system," Appl. Opt. 12, 62-67 (1973).

13. R. M. A. Azzam and N. M. Bashara, "Parametric equation of the locus of invariant-ellipticity states of an optical system," Appl. Opt. 12, 2545-2547 (1973). 